\title{
Generation of coherent two-color pulses at two adjacent harmonics in a seeded free-electron laser
}

\author{
Zhouyu Zhao, Heting Li, ${ }^{*}$ and Qika Jia \\ National Synchrotron Radiation Laboratory, University of Science and Technology of China, \\ Hefei 230029, Anhui, People's Republic of China
}

(Received 24 October 2017; published 9 February 2018)

\begin{abstract}
The growing requirements of pump-probe techniques and nonlinear optics experiments greatly promote the studies of two-color free-electron lasers (FELs). We propose a new method to generate coherent twocolor pulses in a high-gain harmonic generation (HGHG) FEL. In this scheme, an initial tilted electron beam is sent though the modulator and dispersive section of an HGHG FEL to generate the bunching at harmonics of the seed laser. Then a transverse gradient undulator (TGU) is adopted as the radiator and in such radiator, only two separated fractions of the tilted beam will resonate at two adjacent harmonics of the seed laser and are enabled to emit the coherent two-color pulses simultaneously. The time separation between the two pulses are on the order of hundreds of femtoseconds, and can be precisely controlled by varying the tilted amplitude of the electron beam and/or the transverse gradient of the TGU radiator. Numerical simulations confirm the validity and feasibility of this scheme in the extreme ultraviolet waveband.
\end{abstract}

DOI: 10.1103/PhysRevAccelBeams.21.020701

\section{INTRODUCTION}

The short-wavelength free-electron laser (FEL) characterized by high brightness, good coherence, and ultrashort pulse promotes cutting-edge science in atomic physics, chemistry, and biology, etc. For some specific experiments, the output characteristics of FEL pulses both in spectral and temporal domain are required to be adjusted to satisfy the experimental needs. One of the most important formats is to create double FEL pulses which contain two different spectral lines with adjustable time separation, namely, the so-called two-color pulses. Especially, the pump-probe experiments can be accessed by using two-color FEL pulses which open the door for scientists to study the structural dynamics on the atomic and molecular scale.

Recently, the multicolor FEL experiments with different methods have been proposed or carried out in various kinds of radiation frequency band. In summary, there are several schemes to lase at different wavelength, including: (1) Two electron beams with different energy pass through an undulator and resonate at two different wavelengths, as studied both in theory [1] and experiment [2-6]. In this approach, each pulse is independent then the saturation power are similar in condition of maintaining minimum

\footnotetext{
*liheting@ustc.edu.cn

Published by the American Physical Society under the terms of the Creative Commons Attribution 4.0 International license. Further distribution of this work must maintain attribution to the author(s) and the published article's title, journal citation, and DOI.
}

undulator length. However, it is difficult to obtain such beam and the tunability of pulses both in spectral and temporal domain is limited. (2) A single mono-energetic electron beam is used to produce two-color beams by employing two undulator sections with different undulator fields [7-9], or by alternating the undulator segments fields [10]. In the split undulator configurations, the pulse delay can be adjusted by changing the field strength of the delay section. However, since the two-color pulses are generated by the same beam, its saturation power is comparatively lower than that of the first approach. In addition, two-color self-seeded beam can be produced by a single beam using seeding crystal double reflections [11]. (3) A twin-pulseseed laser is adopted to generate two-color FEL radiation with precisely controlled time separation and wavelength, which has been demonstrated at FERMI FEL, one of the most promise two-color FEL sources [12-14]. (4) A new method named the fresh-slice technique has been proposed [15-17] and demonstrated experimentally [15,18]. The beam goes through alternating undulator segments and then laser is generated by different part of the beam. By manipulating the vertical positions of the tail and head of the beam, this approach has the virtue of the central wavelength of each pulse could be adjusted independently, and the pulse separation could be also adjusted precisely. Each pulse can reach saturation as in this method two parts of electron beam are employed for lasing. It allows further flexibility for generating two-color FEL pulses.

Inspired by these works, in this paper we propose to utilize a transverse tilted beam to create two-color FEL radiation based on the high-gain harmonic generation 


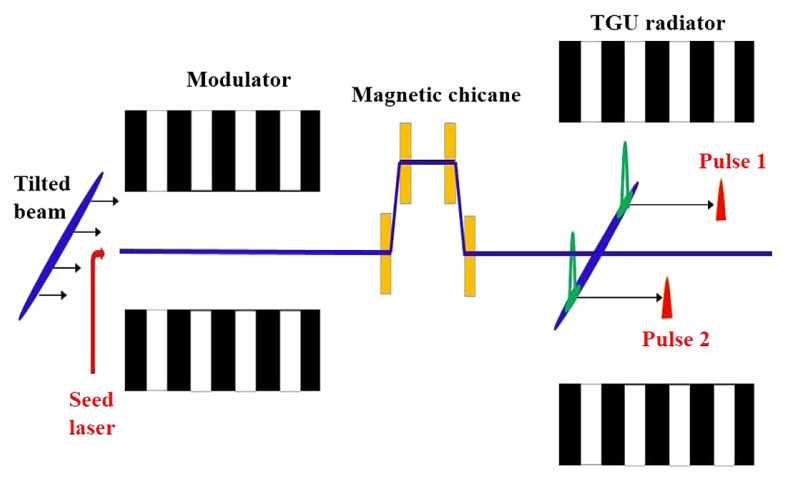

FIG. 1. Schematic of the proposed scheme for the two-color FEL generation.

(HGHG) FEL with a transverse gradient undulator (TGU) radiator. In this method, as shown in Fig. 1, a tilted beam is firstly modulated by a single seed laser and then dispersed by a magnetic chicane. As a result, bunching at harmonics is generated. In the TGU radiator, the electrons at different transverse position will experience different magnetic field and only when the electron energy and the magnetic field resonate at the harmonic of the seed laser, the intense radiation will be generated. Therefore, with the optimization of the tilted amplitude of the electron beam and the gradient of the TGU radiator, this scheme can be contributed to generate two-color pulses at the adjacent harmonics, e.g., one pulse at the 4th harmonic and another at the 5th harmonic. The detailed description of the working principle is given in Sec. II and three-dimension simulations are shown in Sec. III. Several issues are discussed in Sec. IV and finally we summarize in the last section.

\section{METHODS}

The electrons in the so-called "tilted beam" can be characterized by the transverse offset $x$ in proportion to the longitudinal position $s$. The coupling between the longitudinal and transverse positions of the particles is normally unwanted in the conventional FEL facilities for the reason that only a fraction of the beam can contribute to the FEL radiation and therefore the corresponding output performance is degraded. However, the recent researches demonstrated that the tilted beam has many important applications, such as production of broad-bandwidth radiation [19,20], generation of ultrashort FEL pulse [21-23], generation of two-color pulses $[15,18,24,25]$ and so on. Such applications can satisfy some specific experimental requirements, for instance, the pump-probe experiments and nonlinear optics experiments. To obtain such a tilted beam, several methods have been developed, including the rf deflecting cavity which is developed as the electron bunch diagnostic [26-28], transverse wakefields in the corrugated structure [29-33], residual dispersion from compression structure $[34,35]$.
We assume an ideal tilted beam produced by the methods mentioned above with a linear tilted amplitude $\eta=d x / d s$. The tilted amplitude can be tuned in a quite large range. As shown in Fig. 1, the tilted beam travels parallel along the normal modulator axis in which a seed laser is used to modulate the beam. The seed laser radius should be large enough to ensure the adequate energy modulation in each part of the beam.

After the beam passing through a magnetic chicane, the energy modulation is converted into density modulation and bunching at the harmonics is generated.

In a conventional HGHG FEL, then the electron beam and the field of the radiator will resonate at the $n$th harmonic of the seed laser. The radiation wavelength $\lambda_{n}$ can be written as

$$
\lambda_{n}=\frac{\lambda_{u}}{2 \gamma^{2}}\left(1+\frac{K^{2}}{2}\right)=\frac{\lambda_{0}}{n}
$$

where $\gamma$ is the Lorentz factor of the electrons, $\lambda_{0}$ is the wavelength of the seed laser, and $K, \lambda_{u}$ are the strength parameter and period of the radiator, respectively.

However, in the proposed scheme, a transverse gradient undulator (TGU) is adopted as the radiator whose normalized field parameter $K$ has a linear $x$ dependence [36,37],

$$
K(x)=K_{0}(1+\alpha x)
$$

where $K_{0}$ is the dimensionless field strength on the axis of the TGU and $\alpha$ is the field gradient.

In this TGU radiator, different part of the electron beam will experience different undulator magnetic field. The resonant wavelength $\lambda_{r}(s)$ along the whole bunch is

$$
\lambda_{r}(s)=\frac{\lambda_{u}}{2 \gamma^{2}}\left[1+\frac{K^{2}(s)}{2}\right]
$$

One can see that the resonant wavelength from one side to another side (i.e., from the head to the tail) of the beam will become longer or shorter in a quadratic curve.

However, only the radiation at the harmonics of the seed laser will be coherently amplified because the electron beam is bunched at these discrete wavelengths. If we carefully optimize the values of $\eta, K_{0}$ and $\alpha$ to make the resonant wavelengths along the whole bunch covering two adjacent harmonics of the seed laser, two short FEL pulses with different frequencies will be generated from two fractions of the bunch. Due to the limited gain bandwidth, each pulse will also have a short pulse length. The other electrons will experience self-amplified spontaneous emission and contribute little to the total output power.

The time separation between the two FEL pulses is determined by the longitudinal positions of the two bunch fractions that emit the coherent radiation. Combining the coupling relation $x=\eta s$ and Eq. (2), we can get 


$$
\Delta t=\frac{\Delta s}{c}=\frac{\Delta K}{c \alpha \eta K_{0}}
$$

where $c$ is the speed of the light in the vacuum.

Now we assume $\lambda_{1}$ and $\lambda_{2}$ are the radiation wavelengths at the two adjacent harmonics, corresponding to the $n$th and $(n+1)$ th harmonic, respectively. From the resonance condition, we obtain

$$
\Delta K \approx \frac{2 \gamma^{2}}{\lambda_{u}} \frac{1}{K_{\mathrm{aver}}} \frac{\lambda_{0}}{n(n+1)} .
$$

Here $K_{\text {aver }}$ is the average $K$ value for the two radiation wavelengths.

According to Eq. (5), the harmonic order $n$ should not be too small, otherwise the required gradient of the TGU will be too large. In contrast, due to the limited harmonic upconversion efficiency, the harmonic order also cannot be too large. Moreover, large harmonic orders will require a long TGU and lead to a low radiation power. Therefore, the harmonic order $n$ should be moderate.

To make the two-color pulses close to the axis, one should set the $K_{\text {aver }}$ as the undulator strength on the axis of the TGU radiator, namely, $K_{0}=K_{\text {aver }}$. On this condition, the two FEL pulses will locate on both sides of the undulator axis. Inserting Eq. (5) into Eq. (4), we achieve the time separation between the two FEL pulses as

$$
\Delta t \approx \frac{1}{c \alpha \eta K_{0}^{2}} \frac{2 \gamma^{2}}{\lambda_{u}} \frac{\lambda_{0}}{n(n+1)} .
$$

Obviously, the time separation is inversely proportional to $\alpha$ and $\eta$. And based on the relation of $x=\eta s$, the transverse separation of the two pulses can be easily given as

$$
\Delta x \approx \frac{1}{\alpha K_{0}^{2}} \frac{2 \gamma^{2}}{\lambda_{u}} \frac{\lambda_{0}}{n(n+1)} .
$$

\section{SIMULATIONS}

To validate the feasibility of this new method, we have done the three-dimension time-dependent simulations based on the main parameters of Dalian Coherent Light Source (DCLS), which is an extreme ultraviolet (EUV) FEL user facility based on the principle of HGHG-FEL [38]. GENESIS 1.3 code [39] was used to perform the energy modulation in the modulator and two-color FEL generation in the radiator, and ELEGANT code [40] was used to track the phase space evolution in the magnetic chicane.

The electron beam used in the simulations has the following properties: a flattop beam with beam energy of $400 \mathrm{MeV}$, slice energy spread of $20 \mathrm{keV}$, normalized transverse emittance of $1.0 \mathrm{~mm} \cdot \mathrm{mrad}$, peak current of $1 \mathrm{kA}$, full width of $1 \mathrm{ps}$ and a linear tilted amplitude $\eta=11.7$.

The modulator consists of 20 periods with period length of $50 \mathrm{~mm}$. A $240 \mathrm{~nm}$ seed laser with beam waist of $1.5 \mathrm{~mm}$

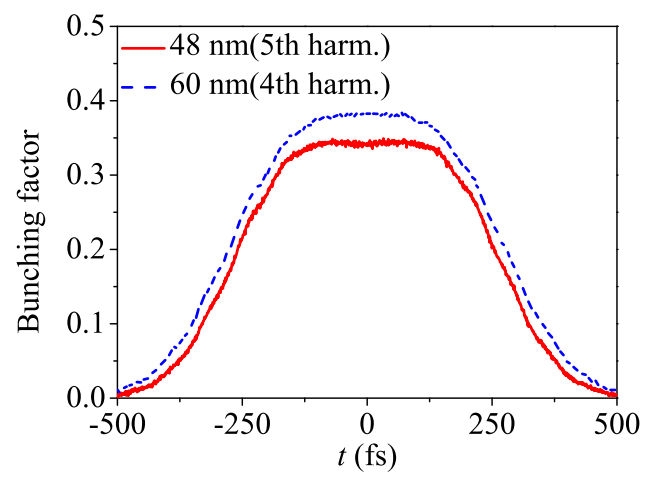

FIG. 2. The 4th and 5th harmonics' bunching factors of the electron beam at the exit of the dispersive section.

is used to modulate the electron beam. Due to the Gaussian distribution of the seed power in the transverse direction, the modulation is nonuniform for the electrons in different transverse positions. The average modulation amplitude is $A=10$, where $A$ means the ratio of maximum energy modulation to the initial local energy spread.

Next, a magnetic chicane follows the modulator to convert the energy modulation into density modulation and then bunching at the harmonics of the seed laser is generated. Then, the bunched beam enters the TGU radiator to emit coherent radiation at the harmonics of the seed laser. We consider to generate the two-color pulses at the 4th and 5th harmonics of the seed laser, i.e., $n=4$ for Eq. (5). The TGU radiator is composed of 50 periods with period length of $30 \mathrm{~mm}$ and transverse gradient of $\alpha=150 \mathrm{~m}^{-1}$. Such short length of the radiator used here benefits from the high-quality electron beam and low harmonic orders converted from the seed laser. In such short modulator and radiator, the beam size varies slowly and therefore it does not have a great influence on the whole process. Actually, the betatron oscillation and the phase advance of the electron beam have been included in the simulations.

The bunching factors for these two harmonics at the entrance of the radiator are shown in Fig. 2. Obviously, the bunching factors also have Gaussian shapes due to the nonuniform energy modulation. Benefitting from such Gaussian distribution, one can adjust the ratio of the pulse energy between the two-color pulses by varying the undulator strength on the center axis, which determines the transverse positions of the two resonant fractions of the electron beam as well as the corresponding bunching factors. Here, to make the two fractions generating the two FEL pulses with considerable bunching factors, as mentioned in the previous section, we set $K_{0}=K_{\text {aver }}=1.5$, while the resonant undulator strengths for the 4th and 5th harmonics are $K=1.7$ and 1.4 , respectively.

The time structure and spectrum of the output FEL pulses at the end of the TGU radiator are given in Fig. 3. For the $48 \mathrm{~nm}$ pulse (5th harmonic), the peak power is about $105 \mathrm{MW}$, the FWHM pulse width is $52.3 \mathrm{fs}$ and the 

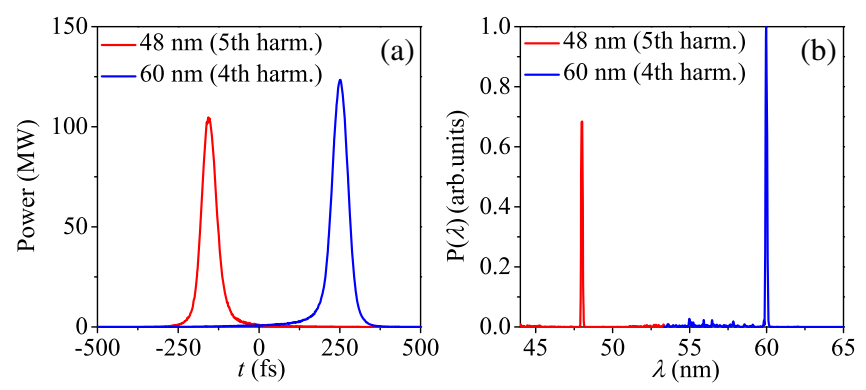

FIG. 3. The time structure (a) and the spectrum (b) of the twocolor FEL pulses at the exit of the TGU radiator.

FWHM bandwidth is about $0.26 \%$, while for the $60 \mathrm{~nm}$ pulse (4th harmonic), the peak power is about $125 \mathrm{MW}$, the FWHM pulse width is 56.8 fs and the FWHM bandwidth is about $0.2 \%$. Both the two pulses are close to Fourier transform limit and the radiation power of the two pulses is very close to each other. Owning to the lower bunching factor and higher harmonic orders, the power of $48 \mathrm{~nm}$ radiation is a little lower than that of $60 \mathrm{~nm}$. The time separation between the two pulses is about $403 \mathrm{fs}$ while it is 415 fs calculated from Eq. (6).

Figure 4 shows the transverse distribution of the twocolor pulses. It can be seen that the two pulses are close to be symmetric about the undulator axis and have a transverse separation of about $1.45 \mathrm{~mm}$ while it is $1.46 \mathrm{~mm}$ calculated from Eq. (7). This spatial separation can be compensated by a carefully designed beam line. In principle, the core of the two pulses can be treated as two spots of a parallel light beam approximately, because the two FEL pulses are of very low emittance. Thus, with a carefully designed reflecting mirror with toroidal surface, two-color pulses can achieve a spatial overlap on the sample. However, the optical path difference induced by the beam line should be considered.

Note that, since the separation of the radiation wavelength is too large to be covered in a single run, the simulations of the FEL radiation in the radiator were done with two separate runs and each run focused on

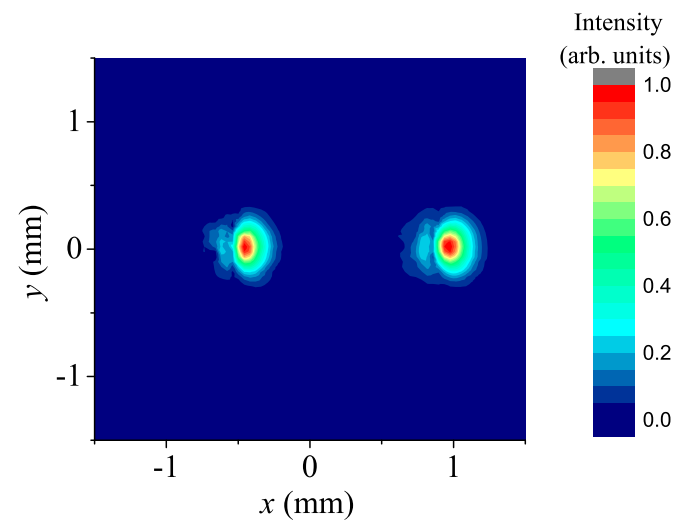

FIG. 4. The transverse distribution of the two-color pulses. one harmonic of the seed laser. As the two FEL pulses are generated from two separated fractions of the electron bunch, these two radiation processes have little impact on each other, therefore we think the simulation results are believable.

\section{DISCUSSIONS}

In the proposed scheme, the generation of the two-color pulses relies upon the tilted beam and the transverse gradient field of the radiator. Concerning these two important factors, several issues should be considered.

The initial tilted beam has a large horizontal size comparing with that in a normal FEL, therefore, a large transverse beam size of the seed laser is required. Considering that the two bunch fractions emitting the two FEL pulses locate in the two sides of the undulator axis symmetrically, the rms transverse beam size $\sigma_{s}$ of the seed laser should be at least larger than the half of the transverse distance of the two pulses, i.e., $\sigma_{s}>\Delta x / 2$. Figure 5 shows the FEL power of the two pulses as a function of the rms radius of the seed laser. The average energy modulation amplitude is fixed to be $A=10$. When $\sigma_{s}<\Delta x / 2$, the power of the two pulses is very low, and when $\sigma_{s}$ grows to 3 times larger than $\Delta x / 2$, the powers of the two pulses do not grow any more with the further increase of the seed radius. However, as given in Eq. (6), the time separation of the two FEL pulses is not affected by the beam size of the seed laser.

Then we consider the tolerances of time separation and the radiation power on the tilted amplitude $\eta$ and the transverse gradient $\alpha$. Figure 6 shows the variations of the time separation and the radiation power of the two-color pulses with the tilted amplitude of the electron beam and the variations with the transverse gradient are given in Fig. 7.

From Figs. 6(a) and 7(a), one can find that the time separation is in inverse proportion to the tilted amplitude and the transverse gradient, and the simulation results are

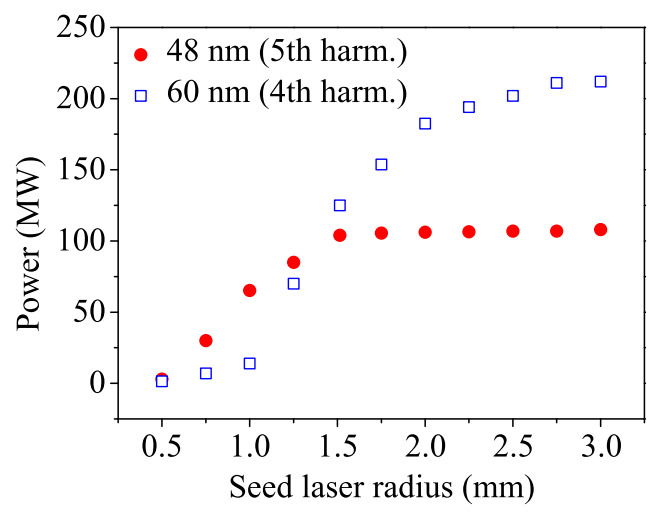

FIG. 5. The FEL power as a function of the rms seed laser radius. The average energy modulation amplitude is fixed to be $A=10$. 

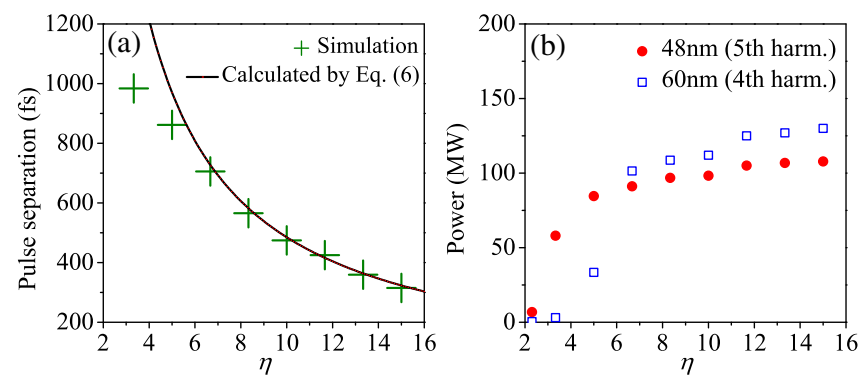

FIG. 6. The variations of the time separation (a) and the radiation power (b) of the two-color pulses with the tilted amplitude $\eta$. The transverse gradient is $\alpha=150 \mathrm{~m}^{-1}$.
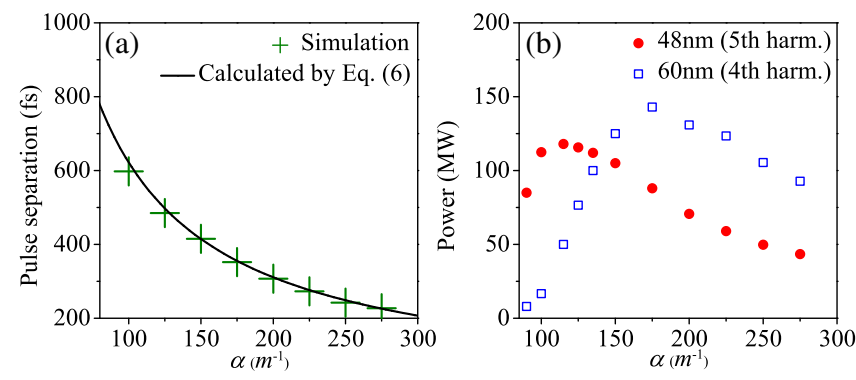

FIG. 7. The variations of the time separation (a) and the radiation power (b) of the two-color pulses with the transverse gradient $\alpha$. The tilted amplitude is $\eta=11.7$.

in good agreement with Eq. (6). Changing the tilted amplitude is more efficient for changing the time separation, because actually the transverse gradient is not easy to be changed for an existing TGU [41], but the tilted amplitude of the electron beam can be conveniently tuned by the methods mentioned in Sec. II. In addition, for a fixed bunch length, increasing the tilted amplitude $\eta$ or the transverse gradient $\alpha$ can make the width of each FEL pulse shorter. From Figs. 6(b) and 7(b), it is clear that the variation of the radiation power is not rapid while changing the tilted amplitude and the transverse gradient in a quite large range.

\section{SUMMARY}

In this paper we have proposed a new method to produce two-color pulses based on HGHG FEL in the EUV waveband. The simulation results based on DCLS parameters have shown that two coherent FEL pulses at the adjacent harmonics of the seed laser can be generated simultaneously from two separated fractions of the electron bunch in the TGU radiator. Each pulse can achieve its own saturation and the ratio of the pulse energy between the two-color pulses can be easily tuned. These two pulses have a time separation of several hundred femtoseconds and a transverse distance of a few millimeters, and each pulse has a ultrashort pulse width of several ten femtoseconds with a bandwidth close to Fourier limit. Both of the temporal and transverse separation can be precisely controlled by adjusting the initial tilted amplitude of the electron beam and/or the transverse gradient of the radiator. Such magnitude of the time separation can directly meet the demand of some of the two-color user experiments.

The FEL scientific community has developed several schemes to produce two-color FEL pulses covering different ranges of photon energies, pulse delays, color separations and pulse durations. In these schemes, the proposed scheme is similar to the methods developed and used for two-color generation at FERMI FEL [12-14], which is based on seeding the same electron beam with two independent laser pulses and on single radiator or splitting the radiator into two subsections. Both schemes are lasing in the UV/EUV waveband and based on the HGHG FEL. Comparing with the significant method carried out by FERMI, the main advantage of the proposed scheme is that only a single seed laser is required and a large frequency separation between the two colors can be obtained without dividing the radiator into two subsections. However, the special required TGU radiator may bring out some inconvenience. Actually, if the saturation length is not too long, the TGU radiator can be replaced by using the natural gradient of normal planar undulator as proposed in Ref. [37]. In addition, our scheme also has a similar characteristic compared with the fresh-slice technique [15-18], which generates the two-color pulses in different undulator section from the different part of a vertical tilted beam. In contrast, we utilize the transverse tilted beam to emit two-color pulses with a large frequency separation in one TGU radiator.

This scheme also has the potential of generating a single ultrashort pulse, i.e., making only one fraction of the electron bunch resonate at one harmonic of the seed laser. For this case, a larger tilted amplitude will make the single FEL pulse shorter. It also can be used to generate multicolor pulses, however, a large transverse gradient will be required and may be very challenging.

\section{ACKNOWLEDGMENTS}

This work is supported by the National Natural Science Foundation of China under Grants (No. 11675178 and 11205156), and National Key Research and Development Program of China (No. 2016YFA0401901).

[1] F. Ciocci, G. Dattoli, S. Pagnutti, A. Petralia, E. Sabia, P. L. Ottaviani, M. Ferrario, F. Villa, and V. Petrillo, Two Color Free-Electron Laser and Frequency Beating, Phys. Rev. Lett. 111, 264801 (2013).

[2] E. Chiadroni, A. Bacci, M. Bellaveglia et al., The $\mathrm{THz}$ radiation source at SPARC, J. Phys. Conf. Ser. 357, 012034 (2012).

[3] V. Petrillo, M. P. Anania, M. Artioli et al., Observation of Time-Domain Modulation of Free-Electron-Laser Pulses 
by Multipeaked Electron-Energy Spectrum, Phys. Rev. Lett. 111, 114802 (2013).

[4] C. Ronsivalle, M. P. Anania, A. Bacci et al., Largebandwidth two-color free-electron laser driven by a comb-like electron beam, New J. Phys. 16, 033018 (2014).

[5] E. Chiadroni, M. P. Anania, M. Artioli et al., Two color FEL driven by a comb-like electron beam distribution, Phys. Procedia 52, 27 (2014).

[6] A. Marinelli, D. Ratner, A. A. Lutman et al., High-intensity double-pulse X-ray free-electron laser, Nat. Commun. 6, 6369 (2015).

[7] A. A. Lutman, R. Coffee, Y. Ding, Z. Huang, J. Krzywinski, T. Maxwell, M. Messerschmidt, and H.-D. Nuhn, Experimental Demonstration of Femtosecond Two-Color X-ray Free-Electron Lasers, Phys. Rev. Lett. 110, 134801 (2013).

[8] T. Hara, Y, Inubushi, T. Katayama et al., Two-colour hard $\mathrm{X}$-ray free-electron laser with wide tunability, Nat. Commun. 4, 2919 (2013).

[9] A. A. Lutman, J. P. MacArthur, M. Ilchen et al., Polarization control in an X-ray free-electron laser, Nat. Photonics 10, 468 (2016).

[10] A. Marinelli, A. A. Lutman, J. Wu, Y. Ding, J. Krzywinski, H.-D. Nuhn, Y. Feng, R. N. Coffee, and C. Pellegrini, Multicolor Operation and Spectral Control in a GainModulated X-ray Free-Electron Laser, Phys. Rev. Lett. 111, 134801 (2013).

[11] A. A. Lutman, F. J. Decker, J. Arthur et al., Demonstration of Single-Crystal Self-Seeded Two-Color X-ray FreeElectron Lasers, Phys. Rev. Lett. 113, 254801 (2014).

[12] E. Allaria, F. Bencivenga, R. Borghes et al., Two-colour pump-probe experiments with a twin-pulse-seed extreme ultraviolet free-electron laser, Nat. Commun. 4, 2476 (2013).

[13] E. Ferrari, Seeded multicolor FEL pulses: Status and future plans, Synchrotron Radiat. News 29, 4 (2016).

[14] E. Ferrari, C. Spezzani, F. Fortuna et al., Widely tunable two-colour seeded free-electron laser source for resonantpump resonant-probe magnetic scattering, Nat. Commun. 7, 10343 (2016).

[15] A. A. Lutman, T. J. Maxwell, J. P. MacArthur et al., Freshslice multicolour X-ray free-electron lasers, Nat. Photonics 10, 745 (2016).

[16] C. Feng and H. Deng, X-ray lasers: Multicolour emission, Nat. Photonics 10, 695 (2016).

[17] W. Qin, Y. Ding, A. A. Lutman, and Y.-C. Chao, Matchingbased fresh-slice method for generating two-color x-ray free-electron lasers, Phys. Rev. Accel. Beams 20, 090701 (2017).

[18] C. Emma, A. Lutman, M. W. Guetg, J. Krzywinski, A. Marinelli, J. Wu, and C. Pellegrini, Experimental demonstration of fresh bunch self-seeding in an X-ray free electron laser, Appl. Phys. Lett. 110, 154101 (2017).

[19] E. Prat, M. Calvi, and S. Reiche, Generation of ultra-largebandwidth $\mathrm{x}$-ray free-electron-laser pulses with a transversegradient undulator, J. Synchrotron Radiat. 23, 874 (2016).

[20] A. S. Hernandez, E. Prat, S. Bettoni, B. Beutner, and S. Reiche, Generation of large-bandwidth x-ray free-electronlaser pulses, Phys. Rev. Accel. Beams 19, 090702 (2016).

[21] P. Emma and Z. Huang, Femtosecond x-ray pulses from a spatially chirped electron bunch in a SASE FEL, Nucl. Instrum. Methods Phys. Res., Sect. A 528, 458 (2004).
[22] E. Prat, F. Lohl, and S. Reiche, Efficient generation of short and high-power x-ray free-electron-laser pulses based on superradiance with a transversely tilted beam, Phys. Rev. ST Accel. Beams 18, 100701 (2015).

[23] H. Li and Q. Jia, Generation of a few femtoseconds pulses in seeded FELs using a seed laser with small transverse size, Nucl. Instrum. Methods Phys. Res., Sect. A 830, 309 (2016).

[24] S. Reiche and E. Prat, Two-color operation of a freeelectron laser with a tilted beam, J. Synchrotron Radiat. 23, 869 (2016).

[25] E. Prat, S. Bettoni, and S. Reiche, Enhanced x-ray freeelectron-laser performance from tilted electron beams, Nucl. Instrum. Methods Phys. Res., Sect. A 865, 1 (2016).

[26] D. Alesini, G. Di Pirro, L. Ficcadenti, A. Mostacci, L. Palumbo, J. Rosenzweig, and C. Vaccarezza, RF deflector design and measurements for the longitudinal and transverse phase space characterization at SPARC, Nucl. Instrum. Methods Phys. Res., Sect. A 568, 488 (2006).

[27] C. Behrens, F. J. Decker, Y. Ding et al., Few-femtosecond time-resolved measurements of $\mathrm{x}$-ray free-electron lasers, Nat. Commun. 5, 3762 (2014).

[28] J. H. Tan, Q. Gu, W. C. Fang, D. C. Tong, and Z. T. Zhao, $\mathrm{X}$-band deflecting cavity design for ultra-short bunch length measurement of SXFEL at SINAP, Nuclear Science and Techniques 25, 060101 (2014).

[29] A. Novokhatski, Wakefield potentials of corrugated structures, Phys. Rev. ST Accel. Beams 18, 104402 (2015).

[30] Z. Zhang, K. Bane, Y. Ding, Z. Huang, R. Iverson, T. Maxwell, G. Stupakov, and L. Wang, Electron beam energy chirp control with a rectangular corrugated structure at the Linac Coherent Light Source, Phys. Rev. ST Accel. Beams 18, 010702 (2015).

[31] K. Bane, G. Stupakov, and I. Zagorodnov, Analytical formulas for short bunch wakes in a flat dechirper, Phys. Rev. Accel. Beams 19, 084401 (2016).

[32] S. Bettoni, P. Craievich, A. A. Lutman, and M. Pedrozzi, Temporal profile measurements of relativistic electron bunch based on wakefield generation, Phys. Rev. Accel. Beams 19, 021304 (2016).

[33] J. Zemella, K. Bane, A. Fisher et al., Measurements of wake-induced electron beam deflection in a dechirper at the Linac Coherent Light Source, Phys. Rev. Accel. Beams 20, 104403 (2017).

[34] M. W. Guetg, B. Beutner, E. Prat, and S. Reiche, Optimization of free electron laser performance by dispersionbased beam-tilt correction, Phys. Rev. ST Accel. Beams 18, 030701 (2015).

[35] E. Prat and M. Aiba, General and efficient dispersion-based measurement of beam slice parameters, Phys. Rev. ST Accel. Beams 17, 032801 (2014).

[36] Z. Huang, Y. Ding, and C. B. Schroeder, Compact X-ray Free-Electron Laser from a Laser-Plasma Accelerator Using a Transverse-Gradient Undulator, Phys. Rev. Lett. 109, 204801 (2012).

[37] Q. Jia and H. Li, Normal planar undulators doubling as transverse gradient undulators, Phys. Rev. Accel. Beams 20, 020707 (2017).

[38] T. Zhang, H.-X. Deng, W.-Q. Zhang, G.-R. Wu, D.-X. Dai, D. Wang, X.-M. Yang, and Z.-T. Zhao, FEL polarization 
control studies on Dalian coherent light source, Chin. Phys. C 37, 118101 (2013).

[39] S. Reiche, GENESIS 1.3: A fully 3D time-dependent FEL simulation code, Nucl. Instrum. Methods Phys. Res., Sect. A 429, 243 (1999).

[40] M. Borland, Argonne National Lab Technical Report No. LS-287, 2000.
[41] J. Li, H. Li, Q. Jia, and B. Du, Calculation and Analysis of the Magnetic Field of a Transverse Gradient Undulator, in Proceedings of the 7th International Particle Accelerator Conference, Busan, Korea, 2016 (JACoW, Busan, Korea, 2016), p. 1130. 